\title{
The structure of claw-free graphs
}

\author{
Maria Chudnovsky and Paul Seymour
}

\begin{abstract}
A graph is claw-free if no vertex has three pairwise nonadjacent neighbours. At first sight, there seem to be a great variety of types of claw-free graphs. For instance, there are line graphs, the graph of the icosahedron, complements of triangle-free graphs, and the Schläfli graph (an amazingly highly-symmetric graph with 27 vertices), and more; for instance, if we arrange vertices in a circle, choose some intervals from the circle, and make the vertices in each interval adjacent to each other, the graph we produce is claw-free. There are several other such examples, which we regard as "basic" claw-free graphs.

Nevertheless, it is possible to prove a complete structure theorem for clawfree graphs. We have shown that every connected claw-free graph can be obtained from one of the basic claw-free graphs by simple expansion operations. In this paper we explain the precise statement of the theorem, sketch the proof, and give a few applications.
\end{abstract}

\section{Introduction}

A graph is claw-free if no vertex has three pairwise nonadjacent neighbours. (Graphs in this paper are finite and simple.) Line graphs are claw-free, and it has long been recognized that claw-free graphs are an interesting generalization of line graphs, sharing some of the same properties. For instance, Minty [16] showed in 1980 that there is a polynomial-time algorithm to find a stable set of maximum weight in a claw-free graph, generalizing the algorithm of Edmonds $[9,10]$ to find a maximum weight matching in a graph.

How do we construct claw-free graphs? Chvátal and Sbihi [8] and Maffray and Reed [15] studied the structure of claw-free perfect graphs, and indeed, it was working on an extension of their results that led us to the present project. But how can we construct the "most general" claw-free graph? This question had not been studied, as far as we know, and yet it turns out to be a very good question. There are several basic types of claw-free graphs, and we were able to show that every connected claw-free graph can be obtained starting from a graph of one of these basic types by means of "expansion" operations (or, in some restricted cases, piecing some of these basic graphs together).

The main goal of this paper is to explain this construction. We are preparing a series of about seven papers containing the results sketched here, but the titles (and the order) of these papers given in the references are provisional, and are currently being revised. In addition, some of the results quoted here are still in the form of notes and have not yet been written down formally, much less been refereed; and while we have tried hard to make sure that the theorems quoted here are true, until they are written down properly we cannot be completely sure. The reader is warned to check with the full published version of these results before relying on them completely.

Before we start to explain the structure theorem, let us introduce "antiprismatic" graphs. We say a graph $G$ is prismatic if for every triangle $T$ of $G$, every vertex of $G$ 
not in $T$ has a unique neighbour in $T$ (a triangle means a 3 -vertex clique). Prismatic graphs have a complex structure, but it turns out that they can be completely described (see sections 6,7 ). We say $G$ is antiprismatic if its complement graph $\bar{G}$ is prismatic. Clearly antiprismatic graphs are claw-free, and they seem to form a subclass of claw-free graphs that is very different from the others. (At least, all our standard methods for analyzing claw-free graphs failed completely when we reached the stage of trying to analyze antiprismatic graphs, and we had to come up with totally new approaches.) Understanding antiprismatic graphs was probably the most difficult part of the project.

The Schläfli graph (a very interesting and highly symmetric graph, described later) is antiprismatic, and it and its induced subgraphs (and some other graphs derived from it by expanding vertices) form a class of antiprismatic graphs, one of (about) eight classes that we need. We showed that every antiprismatic graph either belongs to one of four of these classes, or could be constructed from a sequence of members of the other four classes by repeated hex-joins.

The most important result in this paper is of course the general structure theorem for claw-free graphs. This is explained in detail in sections 3-7, but let us give some idea of it now. We can confine ourselves to connected claw-free graphs $G$, and it is convenient also to assume that $G$ admits no "homogeneous pair of cliques", which we explain in the next section. Then we find that the type of structure that $G$ possesses depends heavily on $\alpha(G)$, the size of the largest stable set of $G$. When $\alpha(G) \geq 4$, it turns out that $G$ is either a kind of generalized line graph, or a circular interval graph. When $\alpha(G)=3$, there are several additional possibilities; for instance, that either $G$ is a subgraph of the icosahedron, or $G$ is expressible as a "hex-join" (explained later), or $G$ is antiprismatic.

Unfortunately the result is rather complicated, and as a warmup we first discuss what are called "quasi-line graphs". A graph $G$ is a quasi-line graph if for every vertex $v$, the set of neighbours of $v$ can be expressed as the union of two cliques. (A clique in $G$ is a set of pairwise adjacent vertices of $G$.) Note that we do not require that two neighbours of $v$ are adjacent if and only if they both belong to one of the cliques; there may be edges between neighbours of $v$ that do not belong to the same clique. Quasi-line graphs are clearly claw-free; they form a proper subset of the set of all claw-free graphs, and a proper superset of the set of all line graphs, and make an interesting half-way stage. We found a structure theorem for all quasi-line graphs, and for that question the answer is much prettier than for general claw-free graphs.

One reason for interest in quasi-line graphs is a problem of Ben Rebea [1]. Since Edmonds' matching algorithm generalizes to claw-free graphs, one might hope that also Edmonds' matching polytope theorem [9] extends to claw-free graphs, and several people $[12,13,14,17]$ have worked on this, although it remains open. We are asking for the list of linear inequalities defining the convex hull of the stable sets of $G$, where we regard a stable set of a $(0,1)$-vector in $\Re^{V(G)}$. For general claw-free graphs $G$ there is not even a conjecture, but Ben Rebea suggested the same problem for quasi-line graphs, and for that class there is a conjectured answer [17]. We have been able to find the desired list of inequalities for all connected quasi-line graphs that are not of one particular type, graphs we call "fuzzy circular interval graphs"; and in a still more recent paper, Eisenbrand, Oriolo, Stauffer and Ventura 
solved the case that we left open. This is explained in section 2 .

For a graph $G$, let $\chi(G), \omega(G)$ denote the chromatic number of $G$ and the maximum size of a clique of $G$. Another result that we might hope to extend from line graphs to general claw-free graphs is Vizing's theorem [18], which implies that if $G$ is a line graph (of a simple graph), then $\chi(G) \leq \omega(G)+1$. For general claw-free graphs (or even for line graphs of non-simple graphs) this is false, although Shannon's theorem implies that $\chi(G)<\frac{3}{2} \omega(G)+1$ for line graphs. Note also that for a $n$-vertex graph $G$ with $\alpha(G) \leq 2$, even the linear bound is false; $\chi(G) \geq n / 2$, and yet $\omega(G)$ may be $o(n)$. Nevertheless, for connected claw-free graphs with $\alpha(G) \geq 3$, the structure is much more controlled, and we were able to show that for any such graph, $\chi(G) \leq 2 \omega(G)$ (and this is asymptotically best possible). We discuss colouring claw-free graphs in section 8 .

\section{Quasi-line graphs}

Construct a graph $G$ as follows. Take a circle $C$, and let $V(G)$ be a finite set of points of $C$. Take a set of intervals from $C$ (an interval means a proper subset of $C$ homeomorphic to $[0,1])$; and say that $u, v \in V(G)$ are adjacent in $G$ if $\{u, v\}$ is a subset of one of the intervals. We call such a graph a circular interval graph. All such graphs are claw-free, and indeed they are quasi-line graphs, as is easily seen. (These are a subclass of class of circular arc graphs; they are sometimes called "proper" circular arc graphs.) Linear interval graphs are defined in the same way, taking $C$ to be a line instead of a circle. Every linear interval graph is also a circular interval graph.

There is another way to construct quasi-line graphs, that we explain next. A vertex $v \in V(G)$ is simplicial if the set of neighbours of $v$ is a clique. A strip $(G, a, b)$ consists of a claw-free graph $G$ together with two designated simplicial vertices $a, b$ called the ends of the strip. For instance, if $G$ is a linear interval graph, with vertices $v_{1}, \ldots, v_{n}$ in order and with $n>1$, then $v_{1}, v_{n}$ are simplicial, and so $\left(G, v_{1}, v_{n}\right)$ is a strip, called a linear interval strip.

Suppose that $(G, a, b)$ and $\left(G^{\prime}, a^{\prime}, b^{\prime}\right)$ are two strips. We compose them as follows. Let $A, B$ be the set of vertices of $G \backslash\{a, b\}$ adjacent in $G$ to $a, b$ respectively, and define $A^{\prime}, B^{\prime}$ similarly. Take the disjoint union of $G \backslash\{a, b\}$ and $G^{\prime} \backslash\left\{a^{\prime}, b^{\prime}\right\}$; and let $H$ be the graph obtained from this by adding all possible edges between $A$ and $A^{\prime}$ and between $B$ and $B^{\prime}$. Then $H$ is claw-free.

This method of composing two strips is symmetrical between $(G, a, b)$ and $\left(G^{\prime}, a^{\prime}, b^{\prime}\right)$, but we do not use it in a symmetrical way. We use it as follows. Start with a graph $G_{0}$ with an even number of vertices and which is the disjoint union of complete graphs, and pair the vertices of $G_{0}$. Let the pairs be $\left(a_{1}, b_{1}\right), \ldots,\left(a_{n}, b_{n}\right)$, say. For $i=1, \ldots, n$, let $\left(G_{i}^{\prime}, a_{i}^{\prime}, b_{i}^{\prime}\right)$ be a strip. For $i=1, \ldots, n$, let $G_{i}$ be the graph obtained by composing $\left(G_{i-1}, a_{i}, b_{i}\right)$ and $\left(G_{i}^{\prime}, a_{i}^{\prime}, b_{i}^{\prime}\right)$; then the resulting graph $G_{n}$ is called a composition of the strips $\left(G_{i}^{\prime}, a_{i}^{\prime}, b_{i}^{\prime}\right)(1 \leq i \leq n)$. For instance, if for each of the strips $\left(G_{i}^{\prime}, a_{i}^{\prime}, b_{i}^{\prime}\right), G_{i}^{\prime}$ is a 3 -vertex path with ends $a_{i}^{\prime}, b_{i}^{\prime}$, then the effect of composing with $\left(G_{i}^{\prime}, a_{i}^{\prime}, b_{i}^{\prime}\right)$ is the identification of $a_{i}, b_{i}$; and so the graphs that are compositions of such 3 -vertex path strips are precisely line graphs.

It is easy to check that every graph that is the composition of linear interval strips is a quasi-line graph, so this gives us a second construction for quasi-line 
graphs (and this includes line graphs, since the 3-vertex strip mentioned above is a linear interval strip). This is not quite the whole story for quasi-line graphs yet; we need one more concept.

A homogeneous pair of cliques in $G$ is a pair $(A, B)$ such that:

- $A, B$ are cliques in $G$ and $A \cap B=\emptyset$,

- no vertex of $G \backslash(A \cup B)$ has both a neighbour and a non-neighbour in $A$, and the same for $B$, and

- either $|A| \geq 2$ or $|B| \geq 2$.

Now we can state one version of our structure theorem for quasi-line graphs, the following.

2.1 For every quasi-line graph $G$, if $G$ is connected and there is no homogeneous pair of cliques in $G$, then either $G$ is a circular interval graph, or $G$ is a composition of linear interval strips.

One might object that this is not quite a structure theorem for all quasi-line graphs. The hypothesis that $G$ is connected is unobjectionable, because if we understand the possibilities for the connected components of a quasi-line graph, then we understand the possibilities for the entire graph; but the same is not true for the "homogeneous pair" hypothesis. Suppose that we wish to understand the structure of a connected quasi-line graph that does admit a homogeneous pair of cliques. We could delete all except one vertex from both of the cliques, and iterate (if there is still a homogeneous pair of cliques, do it again), until 2.1 can be applied; and then add back in all the homogeneous pairs we deleted. But it is not so easy to see how to describe the global structure that results. Below we give a more explicit description.

First, we need to extend the concept of a circular interval graph. We say that a graph $G$ is a fuzzy circular interval graph if:

- there is a map $\phi$ from $V(G)$ to a circle $C$ (not necessarily injective), and

- there is a set of intervals from $C$, none including another, and such that no point of $C$ is an end of more than one of the intervals, so that

- for $u, v$ in $G$, if $u, v$ are adjacent then $\{u, v\}$ is a subset of one of the intervals, and if $u, v$ are nonadjacent then $u, v$ are both ends of any interval including both of them (and in particular, if $\phi(u)=\phi(v)$ then $u, v$ are adjacent).

(If also we required $\phi$ to be injective, this would be equivalent to the definition of a circular interval graph.) If $x, y$ are ends of an interval and one of the sets $\phi^{-1}(x), \phi^{-1}(y)$ has at least two members, then the pair $\left(\phi^{-1}(x), \phi^{-1}(y)\right)$ is a homogeneous pair of cliques; and these turn out to be the only kinds of homogeneous pairs of cliques that we need. (Fuzzy linear interval strips are defined analogously, with the proviso that if $a, b$ are the ends of the strip then $\phi(a), \phi(b)$ are different from $\phi(v)$ for all other vertices $v$ of $G$.) The following is a more explicit version of the structure theorem for quasi-line graphs. 
2.2 For every quasi-line graph $G$, if $G$ is connected, then either $G$ is a fuzzy circular interval graph, or $G$ is a composition of fuzzy linear interval strips.

The current proof of 2.2 is indirect. First we apply the general structure theorem for claw-free graphs, described later; this tells us that our quasi-line graph belongs to one of several basic classes, or is an expansion of such a graph. Then we examine each of these classes separately, to figure out which quasi-line graphs it contains. The most difficult class is the one where we get least information, the class of graphs $G$ with $\alpha(G) \leq 2$; these graphs are always claw-free, but are not necessarily quasi-line graphs, and perhaps half or more of the entire proof is spent on this case. We omit further details, which will appear in a separate paper [6].

Let us turn to the application to Ben Rebea's question, mentioned in the introduction. Let $G$ be a graph, and for $X \subseteq V(G)$, let $\underline{X} \in \Re^{V(G)}$ be the vector where for $v \in V(G), \underline{X}_{v}=1$ if $v \in X$ and $\underline{X}_{v}=0$ if $v \notin X$. The stable set polytope of $G$ is the convex hull of all the vectors $\underline{X}$ such that $X \subseteq V(G)$ is stable. Every point $p$ of the stable set polytope satisfies the following inequalities:

- $p_{v} \geq 0$ for all $v \in V(G)$

- $\sum_{v \in K} p_{v} \leq 1$ for every clique $K$ of $G$

- for every odd list $K_{1}, \ldots, K_{2 n+1}$ of cliques of $G$, if $Y$ denotes the set of all vertices in at least two of $K_{1}, \ldots, K_{2 n+1}$, then $\sum_{v \in Y} p_{v} \leq n$.

(To see this, note that since all the inequalities are linear, it suffices to check that they holds when $p=\underline{X}$ for a stable set $X$.) Let us call these Edmonds' inequalities. When $G$ is a line graph, Edmonds' matching polytope theorem [9] asserts that a point $p \in \Re^{V(G)}$ belongs to the stable set polytope if and only if it satisfies Edmonds' inequalities. For general claw-free graphs this is not true, but the problem seems more tractable for quasi-line graphs, and in [17] there is a conjecture presenting an alternative list of inequalities that may be necessary and sufficient when $G$ is a quasi-line graph. We were able to show the following:

2.3 Let $G$ be a connected quasi-line graph, that is not a fuzzy circular interval graph. Then a point $p \in \Re^{V(G)}$ belongs to the stable set polytope if and only if it satisfies Edmonds' inequalities.

To prove this, we observe first that 2.2 implies that $G$ is a composition of fuzzy linear interval strips. Each of the strips can be adequately simulated by an appropriate 5-vertex strip; if we make the corresponding composition of these simplified strips, we obtain a line graph, for which Edmonds' theorem gives the stable set polytope; and now we replace the simplified strips by the original strips, one by one, and check the effect on the stable set polytope at each step. We omit further details.

More recently, Eisenbrand, Oriolo, Stauffer and Ventura [11] have solved the same question for the case that was still open, for fuzzy circular interval graphs, and hence completed the answer to Ben Rebea's question. 


\section{Claw-free graphs with $\alpha(G) \geq 4$}

Let us begin on the structure theorem for general claw-free graphs. In this section we consider the case of claw-free graphs $G$ with $\alpha(G) \geq 4$. One way to make such a graph is to take the disjoint union of two claw-free graphs $G_{1}, G_{2}$ neither of which is complete, and we cannot hope yet to explain the structure of graphs constructed this way in any finer detail, because at this stage we know nothing about $G_{1}, G_{2}$ (since they might not satisfy $\left.\alpha\left(G_{1}\right), \alpha\left(G_{2}\right) \geq 4\right)$. Thus we had better confine ourselves to connected graphs.

Another way one might try to confound the attempt to describe all connected claw-free graphs $G$ with $\alpha(G) \geq 4$ is the following. For $i=1,2$, let $a_{i}$ be a simplicial vertex of a connected claw-free graph $G_{i}$; and construct $G$ as follows. $G$ is obtained from the disjoint union of $G_{1} \backslash a_{1}$ and $G_{2} \backslash a_{2}$ by making every neighbour of $a_{1}$ in $G_{1}$ adjacent to every neighbour of $a_{2}$ in $G_{2}$. (This is a version of the strip combinations of the previous section, except now we are just using one simplicial vertex instead of two.) The graph $G$ we produce is claw-free and connected (except in trivial cases), and may well satisfy $\alpha(G) \geq 4$, even if $\alpha\left(G_{1}\right) \leq 3$ or $\alpha\left(G_{2}\right) \leq 3$ (or both). So to describe all connected claw-free graphs with $\alpha(G) \geq 4$, we will also need to describe all connected claw-free graphs $G_{1}$ with $\alpha\left(G_{1}\right) \leq 3$ that have a simplicial vertex. Curiously, this can be done; almost all the types of connected claw-free graphs $G_{1}$ with $\alpha\left(G_{1}\right) \leq 3$ cannot have simplicial vertices, and we can explicitly describe those that do. Nevertheless, to simplify the presentation here, we prefer to avoid this step.

Instead, we assume that our graph $G$ cannot be constructed from graphs $G_{1}, G_{2}$ in the way just described. More precisely, let us say that $G$ admits a 1-join if $V(G)$ can be partitioned into four sets $A_{1}, B_{1}, A_{2}, B_{2}$, where $A_{1} \cup A_{2}$ is a clique, $B_{1}, B_{2}$ are nonempty, and there are no edges between $A_{1} \cup B_{1}$ and $A_{2} \cup B_{2}$ except those between $A_{1}, A_{2}$. Except in trivial cases, the claw-free graphs that are expressible as compositions in the way described earlier in this section are precisely the claw-free graphs that admit 1-joins. So henceforth in this section we assume that $G$ does not admit a 1 -join. (Note that this implies that $G$ is connected.)

For such graphs we can describe the structure completely; there is a result analogous to 2.2, except that we need two new kinds of strips, the following:

- Let $G$ be the graph with vertex set $\left\{v_{1}, \ldots, v_{13}\right\}$ and with adjacency as follows. $v_{1}-\cdots-v_{6}$ is a hole in $G$ of length 6 . Next, $v_{7}$ is adjacent to $v_{1}, v_{2} ; v_{8}$ is adjacent to $v_{4}, v_{5} ; v_{9}$ is adjacent to $v_{6}, v_{1}, v_{2}, v_{3} ; v_{10}$ is adjacent to $v_{3}, v_{4}, v_{5}, v_{6}, v_{9} ; v_{11}$ is adjacent to $v_{3}, v_{4}, v_{6}, v_{1}, v_{9}, v_{10} ; v_{12}$ is adjacent to $v_{2}, v_{3}, v_{5}, v_{6}, v_{9}, v_{10}$; and $v_{13}$ is adjacent to $v_{1}, v_{2}, v_{4}, v_{5}, v_{7}, v_{8}$. Let $X \subseteq\left\{v_{11}, v_{12}, v_{13}\right\}$; then the strip $\left(G \backslash X, v_{7}, v_{8}\right)$ is called an $X X$-strip.

- Let $n \geq 0$. Let $A=\left\{a_{0}, a_{1}, \ldots, a_{n}\right\}, B=\left\{b_{0}, b_{1}, \ldots, b_{n}\right\}$ and $C=\left\{c_{1}, \ldots, c_{n}\right\}$ be three cliques, pairwise disjoint. Let $G$ be the graph with vertex set $A \cup B \cup C$ and with adjacency as follows. For $0 \leq i, j \leq n$, let $a_{i}, b_{j}$ be adjacent if and only if $i=j>0$, and for $1 \leq i \leq n$ and $0 \leq j \leq n$ let $c_{i}$ be adjacent to $a_{j}, b_{j}$ if and only if $i \neq j \neq 0$. Let $X \subseteq A \cup B \cup C$ with $a_{0}, b_{0} \notin X$; then the strip $\left(G \backslash X, a_{0}, b_{0}\right)$ is called an antihat strip.

One version of the structure theorem in this case is the following: 
3.1 For every claw-free graph $G$ with $\alpha(G) \geq 4$, if $G$ does not admit a 1-join and there is no homogeneous pair of cliques in $G$, then either $G$ is a circular interval graph, or $G$ is a composition of linear interval strips, $X X$-strips, and antihat strips.

The foregoing is the analogue of 2.1. There is also an analogue of 2.2, using "fuzzy" XX-strips and antihat strips, but we omit the details. The proof of 3.1 is long, about 100 pages or so, and most of it appears in [5]. We sketch some of the proof later in the paper.

\section{The case $\alpha(G)=3$}

What about an analogue of 3.1 for claw-free graphs $G$ with $\alpha(G)=3$ ? There are several extra complications now. First, we have to remember the icosahedron and its induced subgraphs; they are claw-free, and not all accounted for yet. (And there are more graphs of this type to be listed.) Second, and much worse, there are the antiprismatic graphs (they require a couple of sections of their own - see sections 6,7 ). Third, there is another composition operation that shows up now (and the only explanation we see for some of these graphs is that they are compositions of smaller graphs in the same class, so the structure theorem seems to need to use the new composition).

The composition is as follows. For $i=1,2$, let $G_{i}$ be claw-free and non-null, and let $A_{i}, B_{i}, C_{i}$ be disjoint cliques of $G_{i}$ with union $V\left(G_{i}\right)$. Let $G$ be the graph obtained from the disjoint union of $G_{1}, G_{2}$ by making every vertex of $G_{1}$ adjacent to every vertex of $G_{2}$ except that there are no edges between $A_{1}$ and $A_{2}$, between $B_{1}$ and $B_{2}$, and between $C_{1}$ and $C_{2}$. It is easy to see that $G$ is claw-free. We say that $G$ is a hex-join of $G_{1}, G_{2}$. Note that if $G$ admits a hex-join, then the sets $A_{1} \cup B_{2}, B_{1} \cup C_{2}$ and $C_{1} \cup A_{2}$ are three cliques with union $V(G)$, and consequently no graph $G$ with $\alpha(G)>3$ is expressible as a hex-join.

If $V(G)$ is not the union of three cliques (that is, if $\chi(\bar{G}) \geq 4$ ) then $G$ is not expressible as a hex-join; and as for graphs $G$ with $\chi(\bar{G}) \leq 3$, while they might admit hex-joins, the building blocks from which they are made are severely restricted. Thus there is an advantage to handling the two cases $\chi(\bar{G}) \geq 4$ and $\chi(\bar{G}) \leq 3$ separately.

For the first case, we have the following.

4.1 For every claw-free graph $G$ with $\alpha(G) \leq 3$ and $\chi(\bar{G}) \geq 4$, if there is no homogeneous pair of cliques in $G$, then either $G$ is a circular interval graph, or $G$ belongs to the class $\mathcal{S}_{6}$ (defined in the next section), or $G$ is the graph of an XX-strip, or $G$ is a composition of linear interval strips and antihat strips, or $G$ is an induced subgraph of the icosahedron, or $G$ is antiprismatic.

We can tighten up the case when $G$ is a composition of linear interval strips and antihat strips, but we omit those details for simplicity. There is also a "fuzzy" version of this, without the hypothesis that there is no homogeneous pair of cliques in $G$, but it is quite complicated and again we omit it.

If $H$ is a graph, its line graph is denoted by $L(H)$. For the second case, we have:

4.2 For every claw-free graph $G$ with $\chi(\bar{G}) \leq 3$, if there is no homogeneous pair of cliques in $G$ and $G$ admits no hex-join, then either $G$ is a circular interval graph, or a subgraph of $L\left(K_{3, n}\right)$ for some $n$, or the graph of an antihat strip, or antiprismatic. 
There is more work to be done on this yet; this is still a decomposition theorem, and we need to convert it to a structure theorem. We think that every claw-free graph $G$ with $\chi(\bar{G}) \leq 3$ can be built by a series of hex-joins, starting from graphs which are "fuzzy" versions of the graphs of 4.2 ; but these details are not yet worked out.

\section{The decomposition theorem}

To sketch the proofs of 3.1, 4.1 and 4.2, we need another definition. Suppose that $V_{0}, V_{1}, V_{2}$ are disjoint subsets with union $V(G)$, and for $i=1,2$ there are subsets $A_{i}, B_{i}$ of $V_{i}$ satisfying the following:

- for $i=1,2, A_{i}, B_{i}$ are cliques, $A_{i} \cap B_{i}=\emptyset$ and $A_{i}, B_{i}$ and $V_{i} \backslash\left(A_{i} \cup B_{i}\right)$ are all nonempty

- $A_{1}$ is complete to $A_{2}$, and $B_{1}$ is complete to $B_{2}$, and there are no other edges between $V_{1}$ and $V_{2}$, and

- $V_{0}$ is a clique; and for $i=1,2, V_{0}$ is complete to $A_{i} \cup B_{i}$ and anticomplete to $V_{i} \backslash\left(A_{i} \cup B_{i}\right)$.

In this case we say that $G$ admits a generalized 2-join. Define classes $\mathcal{S}_{0}, \ldots, \mathcal{S}_{6}$ as follows.

- $\mathcal{S}_{0}$ is the class of all line graphs.

- $\mathcal{S}_{1}$ is the class of all induced subgraphs of the icosahedron.

- $\mathcal{S}_{2}$ is the class of all graphs of XX-strips.

- $\mathcal{S}_{3}$ is the class of all circular interval graphs.

- $\mathcal{S}_{4}$ is the class of all induced subgraphs of the graph $G$ defined as follows. Let $H$ be the graph with seven vertices $h_{0}, \ldots, h_{6}$, in which $h_{1}, \ldots, h_{6}$ are pairwise adjacent and $h_{0}$ is adjacent to $h_{1}$. Then $G$ is obtained from $L(H)$ by adding one new vertex, adjacent precisely to the members of $V(L(H))=E(H)$ that are not incident with $h_{1}$ in $H$.

- $\mathcal{S}_{5}$ is the class of graphs of antihat strips.

- $\mathcal{S}_{6}$ is the class of all induced subgraphs of the graph $G$ defined as follows. Let $n \geq 0$, and let $V(G)$ be the disjoint union of sets $A, B, C, D$, where $A=$ $\left\{a_{1}, \ldots, a_{n}\right\}, B=\left\{b_{1}, \ldots, b_{n}\right\}, C=\left\{c_{1}, \ldots, c_{n}\right\}$ and $D=\left\{d_{1}, d_{2}, d_{3}, d_{4}, d_{5}\right\}$. The edges of $G$ are as follows.

- $A, B, C$ are cliques

- $a_{i}, b_{i}$ are adjacent for $1 \leq i \leq n$, and $c_{i}$ is adjacent to $a_{j}, b_{j}$ for $1 \leq i, j \leq n$ with $i \neq j$

- $d_{1}$ is completely adjacent to $A \cup B \cup C$; $d_{2}$ is completely adjacent to $A \cup B \cup\left\{d_{1}\right\} ; d_{3}$ is completely adjacent to $A \cup\left\{d_{2}\right\} ; d_{4}$ is completely adjacent to $B \cup\left\{d_{2}, d_{3}\right\}$; and $d_{5}$ is adjacent to $d_{3}, d_{4}$. 
The results 3.1, 4.1 and 4.2 are all consequences of the following decomposition theorem.

5.1 Let $G$ be claw-free and connected. Then either

- $G \in \mathcal{S}_{0} \cup \cdots \cup \mathcal{S}_{6}$, or

- G admits either a homogeneous pair of cliques, a 1-join, a generalized 2-join, or a hex-join, or

- $G$ is antiprismatic.

This is the main theorem of [5]. The proof is very lengthy, about 100 pages. The idea of the proof is, first we choose an appropriate graph $H$, and assume that $H$ is an induced subgraph of $G$; we analyze how the remainder of $G$ can attach to $H$, and infer that either $G$ admits one of the decompositions or falls into one of the classes. Then henceforth we can assume that $G$ does not contain $H$, and we repeat for some other choice of $H$. But making the right sequence of choices for $H$ is crucial, and took a great deal of experiment. The first choice should be that $H$ is the icosahedron; then it is easy to prove that either $G=H$ or $G$ admits one of the decompositions. The same works when $H$ is the icosahedron with one vertex deleted. For technical reasons, it is then best to handle the case when $H$ is the graph of an XX-strip (without the vertices called $v_{11}, v_{12}, v_{13}$ ). Now comes the first major step, that $H$ is a line graph of a cyclically 3 -connected graph, as large as possible; then as long as $H$ is not too small, it is straightforward to analyze the structure of the remainder of $G$ relative to $H$, and we can prove what we want. In particular, this works for "prisms" (two disjoint triangles joined by three disjoint paths) that are not too small, so henceforth we can assume that $G$ contains no such prisms. Next we take $H$ to be the largest induced cycle ("hole") in $G$, and assume it has length at least seven; since $G$ contains no substantial prisms, $G$ now tends to be a circular interval graph (unless it contains some other configurations that we have to handle), so we can assume all holes have length at most 6 . And so on (and it gets worse from here) - we continue through a long series of such steps, assuming that $G$ contains a certain subgraph $H$ but does not contain any subgraph that we already handled. We omit further details.

Using 5.1 to deduce 3.1, 4.1 and 4.2 are rather delicate inductions, and again we omit the details.

\section{Prismatic graphs - the non-orientable case}

The results 3.1, 4.1 and 4.2 reduce the problem to that of understanding antiprismatic graphs. These graphs are very dense, and it seems advantageous now to work in terms of the complement graph; so we want to understand prismatic graphs. We recall that $G$ is prismatic if for every triangle $T$ of $G$, every vertex of $G$ not in $T$ has a unique neighbour in $T$.

If $T_{1}, T_{2}$ are two disjoint triangles of a prismatic graphs $G$, then the edges between $T_{1}$ and $T_{2}$ provide a bijection from $T_{1}$ to $T_{2}$. We say $G$ is orientable if there is a cyclic orientation of each triangle such that for every pair of disjoint triangles, the matching between them preserves the orientation, and non-orientable otherwise. It is helpful 
to divide the problem into two subproblems, the orientable and non-orientable cases. In this section we discuss the non-orientable case.

Before we go on, we need some more definitions. Let us say the core of $G$ is the subgraph induced on the union of all triangles in $G$. If the core is 3-colourable then $G$ is orientable, as is easily seen. Let $G$ be a prismatic graph; here are some constructions that lead to more prismatic graphs. First, let $T$ be a triangle of $G$, say $T=\left\{a_{1}, b_{1}, c\right\}$. We say $T$ is a leaf triangle at $c$ if $a_{1}, b_{1}$ both only belong to one triangle (namely, $T$ ). If this is so, let $H$ be the graph obtained from $G$ by adding new vertices $a_{2}, \ldots, a_{k}, b_{2}, \ldots, b_{k}$, where for $2 \leq i \leq k, a_{i}$ has the same neighbours as $a_{1}$ in $G \backslash\left\{a_{1}, b_{1}, c\right\}$, and similarly $b_{i}$ has the same neighbours as $b_{1}$, and $a_{i}, b_{i}$ are adjacent. Then $H$ is prismatic, and we say it is obtained from $G$ by multiplying a leaf triangle.

Second, let $C$ be the core of $G$, and let $V(G) \backslash V(C)=\left\{a_{1}, \ldots, a_{n}\right\}$. For $1 \leq$ $i \leq n$, let $N_{i}$ be the set of neighbours of $a_{i}$ in $C$. (Thus each $N_{i}$ is stable.) For $1 \leq i \leq n$, let $A_{i}$ be a set of new vertices, and let $H$ be the graph obtained from $C$ by adding $A_{1} \cup \cdots \cup A_{n}$, with adjacency as follows.

- For $1 \leq i \leq n, A_{i}$ is stable.

- For $1 \leq i \leq n$, every vertex in $A_{i}$ has the same neighbours in $C$ as $a_{i}$.

- For $1 \leq i<j \leq n$, if $N_{i} \cap N_{j} \neq \emptyset$ then then there are no edges between $A_{i}, A_{j}$.

- For $1 \leq i<j<k \leq n$, if $\left(N_{i}, N_{j}, N_{k}\right)$ is a partition of $V(C)$ into three stable sets, then there is no triangle in $A_{i} \cup A_{j} \cup A_{k}$.

- Otherwise, adjacency within $A_{1} \cup \cdots \cup A_{n}$ are arbitrary.

(This is not quite as wild as it might appear. For instance, if $i, j, k$ are as in the fourth condition above, then the only restriction on the adjacency between $A_{i}, A_{j}, A_{k}$ is that $A_{i} \cup A_{j} \cup A_{k}$ includes no triangle; none of these pairs of vertices are affected by any of the other conditions, so in a sense the restrictions are "separable". Note also that if $C$ is not 3-colourable, and in particular if $G$ is not orientable, then the fourth condition is vacuously satisfied.) Such a graph $H$ is always prismatic, and we say it is obtained from $G$ by expanding around the core.

If we hope to obtain a structure theorem for all prismatic graphs, the above two constructions will have to be accounted for in it.

Now let us begin on the non-orientable case. Here are two useful prismatic graphs, that we call $P_{1}$ and $P_{2}$.

- $P_{1}$ has nine vertices $a_{1}, a_{2}, a_{3}, b_{1}, b_{2}, b_{3}, c_{1}, c_{2}, c_{3}$, and edges as follows. $\left\{c_{1}, c_{2}, c_{3}\right\}$ is a triangle; for $1 \leq i, j \leq 3, a_{i}$ is adjacent to $b_{j}$; and for $1 \leq i \leq 3, c_{i}$ is adjacent to $a_{i}, b_{i}$.

- $P_{2}$ has ten vertices $x, y, a_{1}, \ldots, a_{8}$, and adjacency as follows. For $1 \leq i \leq 8$, $a_{i}$ is adjacent to $a_{i-1}, a_{i+1}$ and $a_{i+4}$ (reading subscripts modulo 8 ); $x, y$ are adjacent; $x$ is adjacent to $a_{1}, a_{3}, a_{5}, a_{7}$, and $y$ is adjacent to $a_{2}, a_{4}, a_{6}, a_{8}$.

It is not difficult to show the following. 
6.1 Let $G$ be prismatic. Then $G$ is non-orientable if and only if some induced subgraph of $G$ is isomorphic to $P_{1}$ or to $P_{2}$.

To prove this, assign an arbitrary cyclic orientation to each triangle. Make a graph $H$ whose vertices are the triangles of $G$, and whose edges correspond to disjoint pairs of triangles. Each edge of $H$ acquires a sign, depending whether the corresponding matching between the triangles preserves or reverses the cyclic orders of the triangles. If every cycle of $H$ has an even number of orientation-reversing edges, then $G$ is orientable. If not, we examine the shortest cycle of $H$ with an odd number of orientation-reversing edges; it is easy to show that it has length 3 or 4 , and the corresponding three or four triangles of $G$ induce either $P_{1}$ or $P_{2}$.

Let us see some classes of prismatic graphs.

- The Schläfli graph has 27 vertices, and can be described as follows. Let $N$ be the set of all triples $(i, j, k)$ where $1 \leq i, j, k \leq 3$. The vertex set of $G$ is $\left\{a_{i, j, k}:(i, j, k) \in N\right\}$. For distinct $(i, j, k),\left(i^{\prime}, j^{\prime}, k^{\prime}\right) \in N$, let $a_{i, j, k}$ and $a_{i^{\prime}, j^{\prime}, k^{\prime}}$ be adjacent if and only if either

$$
\begin{aligned}
& -k=k^{\prime} \text { and either } i=i^{\prime} \text { or } j=j^{\prime}, \text { or } \\
& -k=k^{\prime}+1(\bmod 3) \text { and } i \neq j^{\prime}, \text { or } \\
& -k=k^{\prime}+2(\bmod 3) \text { and } i^{\prime} \neq j .
\end{aligned}
$$

This graph is much more symmetrical than is apparent from this description — see [2], for instance. (Our thanks to Adrian Bondy, who identified this mysterious graph for us.) The Schläfli graph is antiprismatic, and so all induced subgraphs of its complement are prismatic; we call them 1-prismatic.

- Let $k \geq 0$, and let $G$ have vertex set the disjoint union of five sets $A=$ $\left\{a_{1}, \ldots, a_{k}\right\}, B=\left\{b_{1}, \ldots, b_{k}\right\}, C=\left\{c_{1}, \ldots, c_{k}\right\}, D=\left\{d_{1}, \ldots, d_{k}\right\}$, and $\{p, q, r, s, t\}$, with adjacency as follows.

- $\{p, q, t\}$ and $\{r, s, t\}$ are triangles, and $A, B, C, D$ are stable

- $p$ is completely adjacent to $A \cup B ; q$ is completely adjacent to $C \cup D ; r$ is completely adjacent to $A \cup D ; s$ is completely adjacent to $B \cup C$

- for $1 \leq i \leq k, a_{i} b_{i}, b_{i} c_{i}, c_{i} d_{i}, d_{i} a_{i}$ are edges, and for $1 \leq i, j \leq k$ with $i \neq j, a_{i} c_{j}$ and $b_{i} d_{j}$ are edges.

We say a graph is 2-prismatic if it is an induced subgraph of $G$ for some choice of $k$.

- Let $G$ have vertex set the disjoint union of $\left\{a_{0}, b_{0}, d_{1}, d_{2}, d_{3}\right\}$ and $\left\{a_{i}, b_{i}, c_{i}\right\}(i=$ $1, \ldots, k)$, where $k \geq 3$. Let the edges of $G$ be as follows:

- $a_{0}$ is adjacent to $a_{1}, \ldots, a_{k}$ and to $c_{1}, \ldots, c_{k} ; b_{0}$ is adjacent to $b_{1}, \ldots, b_{k}$ and to $c_{1}, \ldots, c_{k}$

- for $1 \leq i \leq k, c_{i}$ is adjacent to $a_{i}, b_{i}$

$-1 \leq i, j \leq k$ with $i \neq j, a_{i}$ is adjacent to $b_{j}$

- for $i=1,2,3, d_{i}$ is adjacent to $c_{i}$ and to $c_{4}, \ldots, c_{k}$ 
- for $i, j \in\{1,2,3\}$ with $i \neq j, d_{i}$ is adjacent to $a_{j}, b_{j}$.

Any graph that is an induced subgraph of such a graph $G$ is said to be 3prismatic.

- Let $G$ have vertex set the disjoint union of $\{x, y, z\},\left\{a_{1}, \ldots, a_{m}, b_{1}, \ldots, b_{m}\right\}$ and $\left\{c_{1}, \ldots, c_{n}, d_{1}, \ldots, d_{n}\right\}$, where $m, n \geq 2$. Let the edges of $G$ be as follows:

$-x, y, z$ are pairwise adjacent

- $x$ is adjacent to $a_{1}, \ldots, a_{m}, b_{1}, \ldots, b_{m}$, and $y$ is adjacent to $c_{1}, \ldots, c_{n}$, $d_{1}, \ldots, d_{n}$

- $a_{i}, b_{i}$ are adjacent for $1 \leq i \leq m$, and $c_{j}, d_{j}$ are adjacent for $1 \leq j \leq n$

- for $1 \leq i \leq m$ and $1 \leq j \leq n$, the subgraph induced on $\left\{a_{i}, b_{i}, c_{j}, d_{j}\right\}$ is a cycle of length 4 .

Any graph that is an induced subgraph of $G$ is said to be 4-prismatic.

We proved the following.

6.2 Let $G$ be prismatic, containing $P_{1}$ as an induced subgraph. Then $G$ can be obtained from a 1-, 2-, or 3-prismatic graph by multiplying leaf triangles and expanding around the core.

6.3 Let $G$ be prismatic, containing $P_{2}$ as an induced subgraph, and not containing $P_{1}$. Then $G$ can be obtained from a 1- or 4-prismatic graph by multiplying leaf triangles and expanding around the core.

These results are the main theorems of [4]. In view of 6.1 , this solves our problem for the non-orientable case.

\section{Prismatic graphs - the orientable case}

In the orientable case, the graph may be 3 -colourable, and if so then $\bar{G}$ might admit a hex-join. (Remember we are working in the complement now.) To postpone the problems with hex-joins, let us first assume that the core of $G$ is not 3-colourable. We say that $G$ is a cycle of triangles graph if for some $n \geq 5$ with $n=2$ modulo 3 , there are pairwise disjoint stable subsets $X_{1}, \ldots, X_{2 n+1}$ of $V(G)$ with union $V(G)$, such that, reading subscripts modulo $2 n$ :

- for $1 \leq i \leq n$, there is a nonempty subset $\hat{X}_{2 i} \subseteq X_{2 i}$, and at least one of $\hat{X}_{2 i}, \hat{X}_{2 i+2}$ has cardinality 1 ;

- for $i \in\{1, \ldots, 2 n\}$ and all $k$ with $2 \leq k \leq 2 n-2$, let $j \in\{1, \ldots, 2 n\}$ with $j=i+k$ modulo $2 n$ :

(1) if $k=2$ modulo 3 and there exist $u \in X_{i}$ and $v \in X_{j}$, nonadjacent, then either $i, j$ are odd and $k \in\{2,2 n-2\}$, or $i, j$ are even and $u \notin \hat{X}_{i}$ and $v \notin \hat{X}_{j}$;

(2) if $k \neq 2$ modulo 3 then $X_{i}$ is anticomplete to $X_{j}$; 
(Note that $k=2$ modulo 3 if and only if $2 n-k=2$ modulo 3 , so these statements are symmetric between $i$ and $j$.)

- for $1 \leq i \leq n+1, X_{2 i-1}$ is the union of three pairwise disjoint sets $L_{2 i-1}, M_{2 i-1}, R_{2 i-1}$;

- for $1 \leq i \leq n, X_{2 i}$ is anticomplete to $L_{2 i-1} \cup R_{2 i+1} ; X_{2 i} \backslash \hat{X}_{2 i}$ is anticomplete to $M_{2 i-1} \cup M_{2 i+1}$; and every vertex in $X_{2 i} \backslash \hat{X}_{2 i}$ is adjacent to exactly one end of every edge between $R_{2 i-1}$ and $L_{2 i+1}$;

- for $1 \leq i \leq n$, if $\left|\hat{X}_{2 i}\right|>1$ then

(1) $R_{2 i-1}=L_{2 i+1}=\emptyset$;

(2) if $u \in X_{2 i-1}$ and $v \in X_{2 i+1}$, then $u, v$ are nonadjacent if and only if they have the same neighbour in $\hat{X}_{2 i}$;

- for $1 \leq i \leq n$, if $\left|\hat{X}_{2 i}\right|=1$, then

(1) $R_{2 i-1}, L_{2 i+1}$ are matched, and every edge between $M_{2 i-1} \cup R_{2 i-1}$ and $L_{2 i+1} \cup M_{2 i+1}$ is between $R_{2 i-1}$ and $L_{2 i+1}$;

(2) the vertex in $\hat{X}_{2 i}$ is complete to $R_{2 i-1} \cup M_{2 i-1} \cup L_{2 i+1} \cup M_{2 i+1}$;

(3) if $u \in X_{2 i-1}$ and $v \in X_{2 i+1}$ are nonadjacent then $u \in M_{2 i-1} \cup R_{2 i-1}$ and $v \in L_{2 i+1} \cup M_{2 i+1} \mathrm{x}$.

(4) $M_{2 i-1}, \hat{X}_{2 i-2}$ are matched and $M_{2 i+1}, \hat{X}_{2 i+2}$ are matched.

We proved in [3] that:

7.1 Every cycle of triangles graph is prismatic and orientable, and its core is not 3-colourable. Conversely, if $G$ is an orientable prismatic graph, and its core is not 3 -colourable, then $G$ is a cycle of triangles graph (with one exceptional case when $G$ has exactly five triangles).

Here is a sketch of the proof. The first statement is routine checking. For the second, suppose that $G$ is orientable prismatic, and its core is not 3-colourable. Choose an orientation of each triangle as in the definition of "orientable". Every prismatic graph containing $L\left(K_{3,3}\right)$ as an induced subgraph either has 3-colourable core or is not orientable; so $G$ does not contain $L\left(K_{3,3}\right)$. Since also $G$ does not contain $P_{1}$ (since it is orientable), it follows that for every triangle $T$ of $G$, some vertex $w_{T} \in T$ is in no other triangle. Let the other two vertices of $T$ be $u_{T}, v_{T}$, where the orientation of $T$ is the cyclic permutation $u_{T} \mapsto v_{T} \mapsto w_{T} \mapsto u_{T}$. Let $H$ be the digraph formed by the directed edges $\left(u_{T}, v_{T}\right)$ as $T$ ranges over all triangles of $G$. Then as a graph, $H$ has no triangles, and one can show that in every 4-edge path of $H$, the middle vertex is the head of exactly one of the two middle edges. It follows that the structure of $H$ is very restricted. Moreover, $H$ is connected (as a graph, not as a digraph), for otherwise the hypergraph formed by the triangles of $G$ would not be connected and $G$ would therefore have 3-colourable core, a contradiction; and $H$ must have a directed cycle (for otherwise again the core of $G$ would be 3-colourable); and then the theorem follows easily.

Now we turn to the case when the core is 3 -colourable. Let us say that $G$ is triangle-connected if the hypergraph of triangles of $G$ is connected. 
7.2 Let $G$ be prismatic with 3-colourable core, and not triangle-connected. Then there is a partition $\left(C_{1}, \ldots, C_{k}\right)$ of $V(G)$, and a 3-colouring $(X, Y, Z)$ of $V(G)$, with the following properties:

- for $1 \leq i \leq k, C \cap C_{i}$ is nonempty (where $C$ is the core of $G$ ), and every triangle of $G$ is a subset of one of $C_{1}, \ldots, C_{k}$

- for $1 \leq i \leq k$, the hypergraph of all triangles included in $C_{i}$ is connected

- for $1 \leq i<j \leq k$, if $u \in C_{i}$ and $v \in C_{j}$ are adjacent, then either $u \in X$ and $v \in Y$, or $u \in Y$ and $v \in Z$, or $u \in Z$ and $v \in X$

- for $1 \leq i<j \leq k$, if $u \in C_{i}$ and $v \in C_{j}$ are nonadjacent, and either $u \in X$ and $v \in Y$, or $u \in Y$ and $v \in Z$, or $u \in Z$ and $v \in X$, then neither of $u, v$ belongs to $C$.

This tells us that we may add edges between non-core vertices without changing the core, so that for all $i$ with $1 \leq i<k$, the partition $C_{1} \cup \cdots \cup C_{i}, C_{i+1} \cup \cdots \cup C_{k}$ corresponds to a hex-join of $\bar{G}$. Consequently, if we understand triangle-connected prismatic graphs with 3-colourable core, then we may construct all others by taking a sequence of them hooked together as in 7.2.

Let us say a prismatic graph is substantial if for every subset $S \subseteq V(G)$ with $|S| \leq 2$, there is a triangle disjoint from $S$. The prismatic graphs that are not substantial are easy to describe explicitly, but we omit the details. From now on we shall only be concerned with substantial prismatic graphs.

7.3 Let $G$ be prismatic, substantial and triangle-connected, with 3-colourable core, such that its core is not uniquely 3-colourable. Then the core is an induced subgraph of $L\left(K_{3,3}\right)$.

The proof is easy, but we omit it. Since in this case we understand the core completely, it is straightforward to enumerate the possible adjacencies of the non-core vertices, and we omit the details.

There remains the case when the core is uniquely 3-colourable. Let us say that $G$ is a path of triangles graph if it satisfies the same axioms as a cycle of triangles graph, except we make a linear sequence rather than a circular sequence. More precisely, we say that $G$ is a path of triangles graph if for some integer $n \geq 1$ there are pairwise disjoint stable subsets $X_{1}, \ldots, X_{2 n+1}$ of $V(G)$ with union $V(G)$, such that:

- for $1 \leq i \leq n$, there is a nonempty subset $\hat{X}_{2 i} \subseteq X_{2 i} ;\left|\hat{X}_{2}\right|=\left|\hat{X}_{2 n}\right|=1$, and for $0<i<n$, at least one of $\hat{X}_{2 i}, \hat{X}_{2 i+2}$ has cardinality 1 ;

- for $1 \leq i<j \leq 2 n+1$

(1) if $j-i=2$ modulo 3 and there exist $u \in X_{i}$ and $v \in X_{j}$, nonadjacent, then either $i, j$ are odd and $j=i+2$, or $i, j$ are even and $u \notin \hat{X}_{i}$ and $v \notin \hat{X}_{j}$

(2) if $j-i \neq 2$ modulo 3 then either $j=i+1$ or $X_{i}$ is anticomplete to $X_{j}$; 
- for $1 \leq i \leq n+1, X_{2 i-1}$ is the union of three pairwise disjoint sets $L_{2 i-1}, M_{2 i-1}, R_{2 i-1}$, where $L_{1}=M_{1}=M_{2 n+1}=R_{2 n+1}=\emptyset$;

- if $R_{1}=\emptyset$ then $n \geq 2$ and $\left|\hat{X}_{4}\right|>1$, and if $L_{2 n+1}=\emptyset$ then $n \geq 2$ and $\left|\hat{X}_{2 n-2}\right|>1$;

- for $1 \leq i \leq n, X_{2 i}$ is anticomplete to $L_{2 i-1} \cup R_{2 i+1} ; X_{2 i} \backslash \hat{X}_{2 i}$ is anticomplete to $M_{2 i-1} \cup M_{2 i+1}$; and every vertex in $X_{2 i} \backslash \hat{X}_{2 i}$ is adjacent to exactly one end of every edge between $R_{2 i-1}$ and $L_{2 i+1}$;

- for $1<i<n$, if $\left|\hat{X}_{2 i}\right|>1$ then

(1) $R_{2 i-1}=L_{2 i+1}=\emptyset$;

(2) if $u \in X_{2 i-1}$ and $v \in X_{2 i+1}$, then $u, v$ are nonadjacent if and only if they have the same neighbour in $\hat{X}_{2 i}$;

- for $1 \leq i \leq n$, if $\left|\hat{X}_{2 i}\right|=1$, then

(1) $R_{2 i-1}, L_{2 i+1}$ are matched, and every edge between $M_{2 i-1} \cup R_{2 i-1}$ and $L_{2 i+1} \cup M_{2 i+1}$ is between $R_{2 i-1}$ and $L_{2 i+1}$;

(2) the vertex in $\hat{X}_{2 i}$ is complete to $R_{2 i-1} \cup M_{2 i-1} \cup L_{2 i+1} \cup M_{2 i+1}$;

(3) if $u \in X_{2 i-1}$ and $v \in X_{2 i+1}$ are nonadjacent then $u \in M_{2 i-1} \cup R_{2 i-1}$ and $v \in L_{2 i+1} \cup M_{2 i+1}$

(4) if $i>1$ then $M_{2 i-1}, \hat{X}_{2 i-2}$ are matched, and if $i<n$ then $M_{2 i+1}, \hat{X}_{2 i+2}$ are matched.

If $G$ is a 3 -colourable graph with no triangles, we say that $G$ is a whirl. We proved in [3] that:

7.4 Every substantial path of triangles graph is prismatic and triangle-connected, with a uniquely 3-colourable core. Conversely, let $G$ be substantial, prismatic and triangle-connected, with a non-null, uniquely 3-colourable core. Then $G$ is 3-colourable; and either $G$ is a path of triangles graph, or there is a partition $(X, Y)$ of $V(G)$ so that all triangles are subsets of $X$, and $G \mid X$ is a path of triangles graph, and $G \mid Y$ is a whirl, and we can add edges between non-core vertices in $X$ and $Y$ without changing the core, so that the partition $(X, Y)$ corresponds to a hex-join of the complement.

This completes the statement of our structure theorem for claw-free graphs.

\section{Colouring claw-free graphs}

In this section we discuss some aspects of the proof of the following (proved in $[7])$.

8.1 Let $G$ be claw-free and connected, with $\alpha(G) \geq 3$. Then $\chi(G) \leq 2 \omega(G)$.

Note that the theorem is asymptotically best possible, for if $G$ is as in the definition of "2-prismatic graph" in section 6 , then $H$ is obtained from $\bar{G}$ by deleting the vertices $q, r, s, t$, then $H$ is claw-free and connected, $\alpha(H) \geq 3, \omega(H)=k+1$, and $\chi(H)=2 k$. Before we discuss the proof of 8.1, we remark that there is an easy 
proof of the following weakening $(\Delta(G)$ denotes the maximum degree of vertices of $G)$ :

8.2 Let $G$ be claw-free and connected, with $\alpha(G) \geq 3$. Then $\Delta(G) \leq 4(\omega(G)-1)$, and consequently $\chi(G) \leq 4(\omega(G)-1)$.

Proof. The second statement follows from the first by Brook's theorem. For the first we proceed by induction on $|V(G)|$. Let $v$ be a vertex of maximum degree $\Delta(G)$. Since $\alpha(G) \geq 3$ and $G$ is claw-free, there is a vertex different from and nonadjacent to $v$; and consequently we may choose $u$ nonadjacent to $v$ so that $G \backslash u$ is connected. If $\alpha(G \backslash u) \geq 3$, then from the inductive hypothesis, $\Delta(G \backslash u) \leq 4(\omega(G \backslash u)-1)$; and since $\Delta(G)=\Delta(G \backslash u)$ (because $u, v$ are nonadjacent) and $\omega(G \backslash u) \leq \omega(G)$, the desired result would hold. We may assume then that $\alpha(G \backslash u)=2$. Let $N, M$ be the sets of neighbours and nonneighbours of $u$ respectively (thus $N \cup M=V(G) \backslash\{u\}$ ). Since $G$ is connected, there exists $n \in N$. If $x, y \in M$ are nonadjacent, then at least one of $x, y$ is adjacent to $n$, since $\alpha(G \backslash u)=2$; and not both are adjacent to $n$, since otherwise $n$ would have three nonadjacent neighbours, which is impossible since $G$ is claw-free. Thus $n$ is adjacent to exactly one of $x, y$. It follows that the set of neighbours of $n$ in $M$ is a clique, and so is the set of nonneighbours of $n$ in $M$, and therefore $M$ is the union of two cliques. Since $\alpha(G) \geq 3$, there exist $m_{1}, m_{2} \in M$, nonadjacent. Now let $N_{i}$ be the set of all neighbours of $m_{i}$ in $N(i=1,2)$. We have already seen that every vertex of $N$ is adjacent to exactly one of $m_{1}, m_{2}$, and so $N_{1} \cup N_{2}=N$ and $N_{1} \cap N_{2}=\emptyset$. If $x, y \in N_{1}$ are nonadjacent, then $\left\{x, y, n_{2}\right\}$ is stable, contrary to $\alpha(G \backslash u)=2$. Hence $N_{1} \cup\{u\}$ is a clique, and similarly so is $N_{2} \cup\{u\}$. Thus $V(G)$ is the union of four cliques, and $u$ is in two of them, and so $4 \omega(G) \geq|V(G)|+1 \geq \Delta(G)+4$, as required. This proves 8.2.

However, improving the factor of 4 in 8.2 to 2 seems to be very difficult. The only proof we have of 8.1 is an application of the structure theorem for claw-free graphs; and not only is this theorem difficult itself, but also the application is difficult. Here are some of the ideas. There are two easy tricks that we used many times, the following. First, if there is a vertex whose set of neighbours is the union of two cliques, then its degree is at most $2 \omega(G)-2$, and we can delete it and use induction. And quite often there is such a vertex. For instance, if $G$ is expressed as a generalized 2-join, then using the notation of the definition of "generalized 2-join", any vertex in $A_{1}$ has neighbour set the union of two cliques, as is easy to see. Or if $G$ is a circular interval graph, then the neighbour set of every vertex is the union of two cliques. But there does not always exist such a vertex; for instance, in the Schläfli graph there is no such vertex. Every vertex has degree 16 , and yet $\omega(G)=6$.

The second trick is, that we win if $\omega(G) \geq|V(G)| / 4$. For if $|V(G)|$ is even and there is a perfect matching in $\bar{G}$, then $\chi(G) \leq|V(G)| / 2 \leq 2 \omega(G)$; while if $|V(G)|$ is odd then $\omega(G) \geq(|V(G)|+1) / 4$, so if there is a near-perfect matching in $\bar{G}$ then $\chi(G) \leq(|V(G)|+1) / 2 \leq 2 \omega(G)$ as required. So we can assume that $\bar{G}$ contains no perfect or near-perfect matching, and therefore Tutte's maximum matching theorem can be applied, and again it follows easily (but using the hypothesis that $G$ is connected and claw-free and $\alpha(G) \geq 3$; we omit the details) that $\chi(G) \leq 2 \omega(G)$. So we may assume that $V(G)$ is not the union of four cliques. 
These two tricks get us a long way, and suffice to handle non-antiprismatic graphs, but for antiprismatic graphs the proof gets much more complicated and we omit the details.

We mention that an analogous statement holds in the complement, the following.

8.3 Let $G$ be connected and claw-free, with $\alpha(G) \geq 3$. Then $\chi(\bar{G}) \leq 2 \omega(\bar{G})$.

This is also proved in [7]. Again, it is asymptotically best possible; for if $G=$ $L\left(K_{n}\right)$, then $\chi(\bar{G}) \geq n-2$, and $\omega(\bar{G}) \leq n / 2$. Its proof is not an application of the structure theorem for claw-free graphs, however; we found a direct proof. Roughly, the argument is as follows. Choose a tree $T$ of $G$ so that every vertex of $G$ has a neighbour in $T$, with $|V(T)| \leq 2 \alpha(G)$ and such that some three vertices of $T$ are pairwise nonadjacent in $G$. (It is easy to see that this exists.) For every ordered pair $(u, v)$ of adjacent vertices of $T$, let $N_{u, v}$ be the set of all vertices of $G \backslash(X \cup Y)$ that are adjacent to $u$ and not $v$. For each $(u, v), N_{u, v} \cup\{u\}$ is a clique, and every vertex of $G$ belongs to one of these cliques; and so $\chi(\bar{G}) \leq 2|E(T)| \leq 4 \omega(\bar{G})$. In fact we can cover all the vertices of $G$ without using all ordered pairs $(u, v)$, and by carefully choosing the right subset of pairs (plus possibly one extra pair, not an edge of the tree) we show that $\chi(\bar{G}) \leq 2 \omega(\bar{G})$.

\section{Complexity issues}

We have been finding an "explicit construction" for all claw-free graphs, but it is not quite clear what is meant by this. We has better mean more than just a polynomial-time recognition algorithm, for there is an obvious recognition algorithm for claw-free graphs, with running time $O\left(n^{4}\right)$ when the input has $n$ vertices. So what then do we mean by an "explicit construction"? For instance, here is another attempt at an "explicit construction" - given a claw-free graph $G$ that we have already constructed, choose a subset $X \subseteq V(G)$ such that adding a vertex with neighbour set $X$ will not introduce a claw; and add a vertex with neighbour set $X$. We fervently hope that this doesn't count as solving the problem, or else we have been wasting our time; and yet why doesn't it? For one can easily test in polynomial time whether such a set $X$ has the property we require; on what grounds do we exclude this "construction"?

It is our feeling that we should not be permitted to "guess" the set $X$; if we want to use this set $X$ to construct a larger graph, we should inductively have a construction for $(G, X)$, not just for $G$. With that in mind, let us go back through what we proved, and check that we pass this test. We don't, quite; for instance, we allow outselves the operation of making a hex-join. Making a hex-join requires two smaller graphs $G_{1}, G_{2}$ that we already have constructed, and for each of them we need a partition of its vertex set into three cliques. Unfortunately we are currently "guessing" these cliques; if we want to use hex-joins, we should really be constructing quadruples $(G, A, B, C)$ where $A, B, C$ are disjoint cliques with union $V(G)$, and not just constructing $G$. Happily, this can be done. Such a partition $(A, B, C)$ is just a 3 -colouring of $\bar{G}$, and as we have seen, in most cases $\bar{G}$ does not admit a 3 -colouring; when it does, in most cases the 3-colouring is unique; and when it is not unique, we can describe the graph explicitly and list all the 3 -colourings. So that problem can be overcome. 
There is a similar problem with homogeneous pairs, and that one we found more difficult. We believe it is solved, but we are still checking.

\section{Acknowledgements}

This research was performed while Maria Chudnovsky served as a Clay Mathematics Institute Research Fellow. The work of Paul Seymour was partially supported by ONR grant N00014-01-1-0608 and NSF grant DMS-0070912.

\section{References}

[1] A. Ben Rebea, "Étude des stables dans les graphes quasi-adjoints", Ph. D. thesis, Univ. de Grenoble, 1981.

[2] P. Cameron, "Strongly regular graphs", in Selected Topics in Algebraic Graph Theory (eds. L.W. Beineke and R.J. Wilson), Cambridge Univ. Press, , in press.

[3] Maria Chudnovsky and Paul Seymour, "Claw-free graphs I. Three-coloured prismatic graphs", manuscript, 2004.

[4] Maria Chudnovsky and Paul Seymour, "Claw-free graphs II. Non-3-colourable prismatic graphs", manuscript, 2004.

[5] Maria Chudnovsky and Paul Seymour, "Claw-free graphs IV. Sparse decomposition", manuscript, 2003.

[6] Maria Chudnovsky and Paul Seymour, "Claw-free graphs VI. The structure of quasi-line graphs", manuscript, 2004.

[7] Maria Chudnovsky and Paul Seymour, "Claw-free graphs VII. Colouring clawfree graphs", manuscript, 2004.

[8] V. Chvátal and N. Sbihi, "Recognizing claw-free Berge graphs", J. Combinatorial Theory, Ser. B 44 (1988), 154-176.

[9] J. Edmonds, "Maximum matching and a polytope with 0,1-vertices", $J$. Res.Nat. Bur. Standards 69B (1965), 125-130.

[10] J. Edmonds, "Paths, trees and flowers", Canad. J. Math. 17 (1965), 449-467.

[11] F. Eisenbrand, G. Oriolo, G. Stauffer, P. Ventura, "Circular ones matrices and the stable set polytope of quasi-line graphs", manuscript, 2004.

[12] A. Gallucio and A. Sassano, "The rank facets of the stable set polytope for claw-free graphs", J. Combinatorial Theory, Ser. B 69 (1997), 1-38.

[13] R. Giles and L. Trotter, "On stable set polyhedra for $K_{1,3}$-free graphs", $J$. Combinatorial Theory, Ser. B 31 (1981), 313-326.

[14] T.M. Liebling, G. Oriolo, B. Spille and G. Stauffer, "On non-rank facets of the stable set polytope of claw-free graphs and circulant graphs", manuscript, 2004. 
[15] F. Maffray and B. Reed, "A description of claw-free perfect graphs", J. Combinatorial Theory, Ser. B 75 (1999), 134-156.

[16] G.J. Minty, "On maximal independent sets of vertices in claw-free graphs", Journal of Combinatorial Theory, Ser. B 28 (1980), 284-304.

[17] G. Oriolo, "Clique family inequalities for the stable set polytope for quasi-line graphs", in Special Issue of Discrete Applied Mathematics on Stability Problems (eds. V. Lozin and D. de Werra), , in press.

[18] V.G. Vizing, "On an estimate of the chromatic class of a p-graph", Diskret. Analiz. 3 (1964), 25-30.

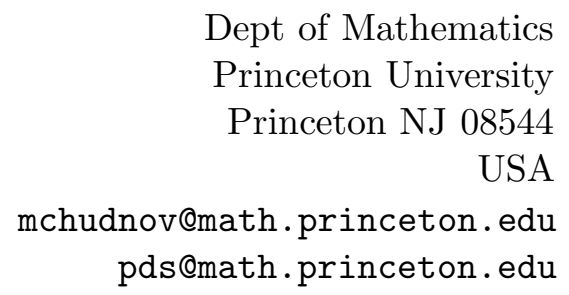

